\title{
Driven nonlinear damping and mode coupling in a superconducting levitated magnet
}

\author{
Xiaodong Jiang $\oplus^{1,2}$ and Mahdi Hosseini ${ }^{1,2,3, *}$ \\ ${ }^{1}$ Elmore Family School of Electrical and Computer Engineering, Purdue University, West Lafayette, Indiana 47907, USA \\ ${ }^{2}$ Birck Nanotechnology Center, Purdue University, West Lafayette, Indiana 47907, USA \\ ${ }^{3}$ Department of Physics and Astronomy, Purdue University, West Lafayette, Indiana 47907, USA
}

(Received 8 September 2021; accepted 25 January 2022; published 18 February 2022)

\begin{abstract}
The magnetic flux pinning phenomenon enables high- $Q$ trapping of a magnet above a superconductor. In practice, the trapped magnet can undergo nonlinear mode coupling and dissipation as its vibration can induce variations of the magnetic field and vortex displacement on the superconductor surface. Here we study nonlinear interactions between vibrational modes of a levitated magnetic mirror above a superconducting disk in vacuum conditions. We observe that by exciting one vibrational mode of the levitated mirror, the vibrational noise of another mode can be suppressed by more than $17 \mathrm{~dB}$. We attribute this unique noise suppression mechanism to the mode coupling and nonlinear dissipation caused by the driven magnetic inhomogeneity of the levitated object. The results suggest that noise reduction of a vibrational mode is possible by driving another orthogonal mode.
\end{abstract}

DOI: 10.1103/PhysRevResearch.4.013132

Levitated mechanical oscillators with exceptionally low mechanical dissipation rates are being investigated for applications in precision sensing and even observation of quantum phenomena at the mesoscopic scale [1]. Observation of quantum effects in acoustic vibrations of nanostructures $[2,3]$ and macroscopic volumes of superfluid liquid [4] are examples where boundaries of quantum and classical mechanics can be studied. Compared with acoustic waves, the center-of-mass motion of micro- and macroscale objects provides a different toolbox to harness the mode coupling and dissipation. Optical tweezers have been proven useful in studying the centerof-mass motion of objects ranging from a single trapped neutral atom [5] to $10^{3}$-atom Bose-Einstein condensates [6] and $10^{8}$-atom solid nanospheres [7]. Other kinds of levitation techniques, such as superconducting levitation, have been proposed for coupling mechanical vibrations to other forms of coherent oscillatory modes [8-10]. Superconducting levitation systems are also being investigated for magnetometry and accelerometry [10-12] and very recently a method for groundstate cooling in these systems was proposed [13]. Although the phenomenon of superconducting levitation in the steady state is well known, the dynamic nature of superconducting trapping and its dissipation mechanisms $[14,15]$ is not well understood.

In this work, we consider a levitated magnetic mirror above a superconductor and study the mode coupling and nonlinear dissipation. We have found that nonlinear coupling between

\footnotetext{
*mh@purdue.edu

Published by the American Physical Society under the terms of the Creative Commons Attribution 4.0 International license. Further distribution of this work must maintain attribution to the author(s) and the published article's title, journal citation, and DOI.
}

different oscillation modes of the mirror can be harnessed to control the damping of certain vibrational modes via an off-resonance drive. For example, by exciting the vertical oscillation mode (along $z$ ) of the magnetic mirror, the magnetic inhomogeneity in the orthogonal directions can be introduced via mode coupling. The orthogonal field fluctuations on the superconducting disk (SD) then lead to enhanced hysteresis losses, damping oscillation energy in modes other than $z$. In this regime, suppressing of vibrational noise of modes orthogonal to $z$ is possible if the excitation frequency is far from the parametric heating regime of the damped modes. The geometry and detection method used in the current levitation experiment enable us to realize such a controlled driven dissipation or "cooling by heating" phenomenon. Here the induced dissipation is the result of mode coupling and nonlinear damping inherent in superconductors.

We use the frozen-image model $[16,17]$ to theoretically calculate the vibrational modes of the levitated magnetic mirror. In this model, we approximate the levitated magnetic mirror with a magnetic dipole $(d)$. This dipole generates one frozen image $\left(d^{\prime}\right)$ before field cooling and then another diamagnetic image $\left(d^{\prime \prime}\right)$ after field cooling inside of the SD during the levitation process, as shown in Fig. 1(b). The magnetic moment $\left(\mathbf{m}_{\text {lev }}\right)$ of the magnetic mirror after levitation makes an angle of $\theta$ with the vertical axis (z), while the initial angle between the magnetic moment $\left(\mathbf{m}_{\text {fro }}\right)$ of the frozen image and $z$ axis is $\theta^{\prime}=90^{\circ}$ before levitation. This is because the magnetization axis of the levitated magnetic mirror is along the $y$ axis in our experiment. The magnetic moment of $d^{\prime \prime}$ is described by $\mathbf{m}_{\text {dia }}$ here.

By calculating the trapping potential energy between the magnetic dipole $(d)$ and these two images $\left(d^{\prime}\right.$ and $\left.d^{\prime \prime}\right)$ and approximating the magnitude of the magnetic moment of the levitated mirror $\left|\mathbf{m}_{\text {lev }}\right|=\left|\mathbf{m}_{\text {fro }}\right|=\left|\mathbf{m}_{\text {dia }}\right|=M$, we can derive the equations of motion of the magnetic mirror along different degrees of freedom. For example, the dynamics of the vertical 
(a)

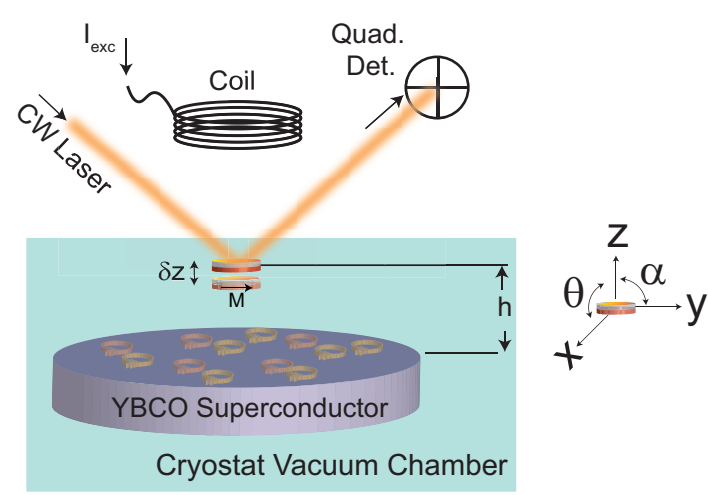

(b)

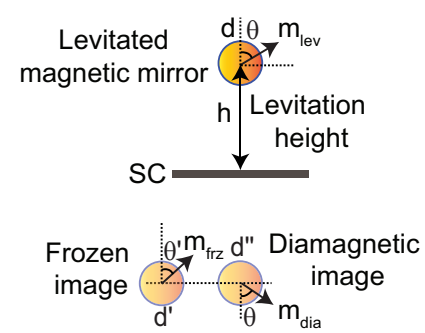

(c)

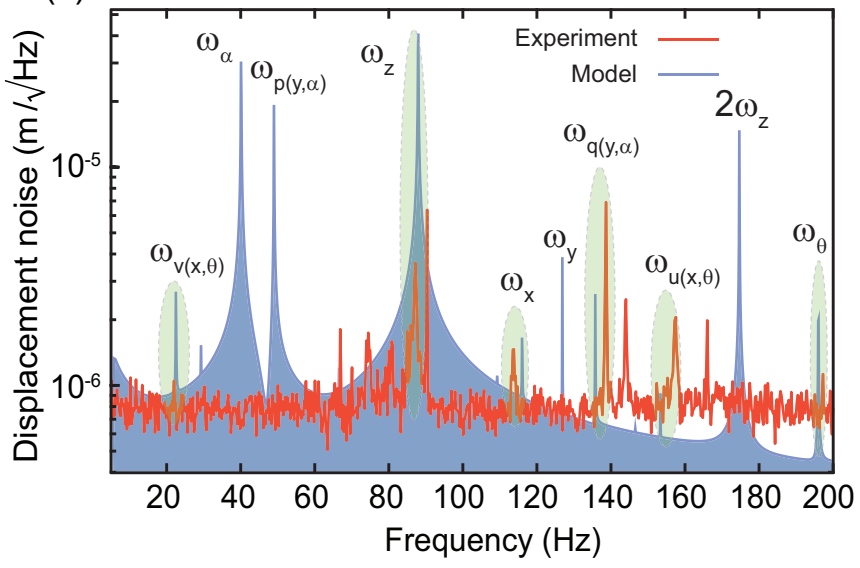

FIG. 1. (a) Experimental setup for a magnetic mirror (a magnetic ring attached to a dielectric mirror) levitated above a superconductor disk and optically probed in vacuum conditions $\left(10^{-8}\right.$ Torr). A coil with excitation current, $I_{\mathrm{exc}}$, can excite and drive the levitated magnet. (b) Frozen-image model diagram. (c) The theoretical (blue) and experimental (red) vibrational noise spectra of the levitated magnetic mirror. The modes whose resonances could be observed (shaded green areas) match the theoretical predictions within $10 \%$. The theoretical spectrum is plotted with an arbitrary offset and excitation.

mode along $z$ axis is given by

$$
\begin{aligned}
& m \ddot{z}+c_{z z} \dot{z}-\frac{3 \mu_{0} M^{2}}{4 \pi}\left\{\frac{3(z+2 h)(\pi / 2-\theta)+x}{\left[x+(z+2 h)^{2}\right]^{5 / 2}}\right. \\
& \left.-\frac{5(z+2 h)^{2}[x+(z+2 h)(\pi / 2-\theta)]}{\left[x+(z+2 h)^{2}\right]^{7 / 2}}-\frac{1}{16(z+h)^{4}}\right\}=m g .
\end{aligned}
$$

That shows the coupling between $z$ and other degrees of freedom, for which similar equations can be derived [18]. Here $M$ is calculated to be $M \simeq 8.4 \times 10^{-4} \mathrm{~A} \mathrm{~m}^{2}, c_{z z}$ is the damping coefficient along $z$ axis, $m=5.3 \times 10^{-6} \mathrm{~kg}$ is the mass of magnetic mirror, $h=1.5 \times 10^{-3} \mathrm{~m}$ is the levitation height, and $g=9.8 \mathrm{~m} / \mathrm{s}^{2}$ is the acceleration of gravity. By numerically solving the coupled equations of motions for different degrees of freedom, we are able to calculate the resonant frequencies of different modes of the levitated magnetic mirror.

The experimental setup is shown in Fig. 1(a). A highreflective $(R>99.8 \%)$ dielectric mirror with the diameter $d=2 \mathrm{~mm}$, mass $m_{r}=3 \mathrm{mg}$, and thickness $t=0.1 \mathrm{~mm}$ is attached to a thin neodymium (Nd) magnetic ring with thickness of $0.05 \mathrm{~mm}$ and mass of $m_{n}=2.3 \mathrm{mg}$. We refer to this combination as the "magnetic mirror." The use of a high-reflective mirror enables precise optical measurements of position and oscillations of the levitated object in different directions. A $\mathrm{YBaCuO} \mathrm{SD}$ with the diameter of $2.5 \mathrm{~cm}$ and thickness of $t=2.5 \mathrm{~mm}$ and critical temperature of $\sim 93 \mathrm{~K}$ is placed inside a closed-cycle and ultra-low-vibration cryostat (DMX-20-OM from Advanced Research Systems) and then cooled down to around $10 \mathrm{~K}$ using cyclic compressed liquid helium. The background gas pressure around the SD and the magnetic mirror was reduced to about $10^{-7}$ Torr. The magnetic mirror is levitated about $h=1.5 \mathrm{~mm}$ above the SD inside the cryostat as shown in Fig. 1(a). A Ti-sapphire laser light is used for its low-noise characteristics and stability at low frequencies, and it is reflected off the magnetic mirror surface and detected by a quadrant detector to sense the mirror's vibrations. To excite the specific mechanical modes of the levitated mirror, we place a 15-turn coil with a diameter of $3 \mathrm{~cm}$ above the mirror and modulate the current in the coil at different frequencies and amplitudes. During measurements the vacuum pump and compressor were temporarily turned off to further reduce the associated mechanical noise.

The relatively large displacement of the levitated magnetic mirror gives rise to the variation of surface current (or diamagnetic image) in the SD that in part changes the trapping potential. As the result, coupling between the translational and rotational modes of the mirror can occur through the SD. We numerically solve the coupled equations of motion and obtain a theoretical vibrational spectrum for the magnetic mirror, as seen in Fig. 1(c). The theoretical spectrum agrees with the experimental data shown in Fig. 1(c). The dominant vibrational mode around $85 \mathrm{~Hz}$ is identified as the oscillation along the vertical direction with the angular frequency of $\omega_{z}$. The coupling between the translational mode $x$ (or $y$ ) and angular mode $\theta$ (or $\alpha$ ) gives rise to the emergence of two coupled modes [16] theoretically found to be $\omega_{v(x, \theta)} \simeq 20 \mathrm{~Hz}$ [or $\left.\omega_{p(y, \alpha)} \simeq 48 \mathrm{~Hz}\right]$ and $\omega_{u(x, \theta)} \simeq 152 \mathrm{~Hz}\left[\omega_{q(y, \alpha)} \simeq 135 \mathrm{~Hz}\right.$ ]. Not all of the modes could be experimentally observed due to the different excitation and detection sensitivity levels of different modes. The slight discrepancy in predicting the frequencies arises from the lack of precise knowledge of the magnet's orientation and its levitation height on thermal cycling. We observe that resonant frequencies of modes can vary slightly every time the SD is recooled. This is because of the change in the stable orientation and position of the magnetic mirror on thermal cycling. This effect has also been observed in Ref. [19] where the resonant frequencies variation as much as $50 \%$ has been reported for superconducting levitated micromagnets. 
To express the magnetic mirror's vibrational amplitude in units of displacement noise, we mount one dielectric mirror on a piezoelectric transducer (PZT) to calibrate the displacement of the levitated magnetic mirror. By keeping the position and detection angle of the quadrant detector the same as in the levitation experiment and then scanning the driving voltage of PZT at $90 \mathrm{~Hz}$, we obtain the vibrational energy of the dielectric mirror as a function of its displacement. In this way, we find the relationship between the quadrant detector's intensity fluctuation and the mirror's displacement. The minimum detectable displacement in our experiment is observed to be $6 \times 10^{-8} \mathrm{~m} / \sqrt{\mathrm{Hz}}$ limited by shot noise and the quadrant detector sensitivity. The optical power in our experiments is chosen to be about $3 \mathrm{~dB}$ above the electronic noise (beyond that the quadrant detector saturates). We also find that the sensitivity of the quadrant detector depends on the light spot size on the detector [20]. By reducing the beam size on the detector we were able to increase the sensitivity by an order of magnitude.

At low background gas pressures and under vacuum conditions, the primary source of mechanical damping of the oscillating magnetic mirror is the inhomogeneity of the mirror's magnetic field distribution giving rise to the hysteretic losses. The field inhomogeneity causes redistribution or density fluctuation of trapped magnetic vortices on the superconductor surface. As the result, energy is dissipated to pay for the altered surface states. The dissipated energy can be quantified by calculating the magnetic field fluctuation $(\delta B)$ on the superconductor surface caused by the oscillations of the levitated magnetic mirror. Similar oscillation-induced dissipation was reported [14] when a macroscopic spherical magnet was levitated above a superconductor under the ambient conditions. The dissipation for different vibrational modes of the levitated magnet strongly depends on the angle between the magnet's magnetization axis $(M)$ and the superconductor surface [14]. A very low dissipation coefficient is expected if the motion of the levitated object is along $M$, when $M$ is parallel to the superconductor surface. When the dissipation due to the background gas and external magnetic field fluctuations are negligible, the quality factors of different vibrational modes are determined by the geometrical factors such as the magnetic inhomogeneity of the levitated object, its magnetic axis, external excitation, and the superconductor configuration. Therefore, we expect different vibrational modes to have different linewidths, which is in agreement with our experimental observations as shown in Fig. 1(c).

The vibrational mode along $z$ axis shows the lowest mechanical $Q$ factor as its motion induces the largest magnetic field inhomogeneity on the superconductor surface. The mechanical $Q$ factor measured at $\omega_{q(y, \alpha)}$ is more than 3000 , limited by the measurement's resolution bandwidth of $0.05 \mathrm{~Hz}$. Using a resolution bandwidth of $0.001 \mathrm{~Hz}$, we could observe $Q=3600$ for the $\omega_{v(x, \theta)}$ mode, which is no longer limited by our measurements [see Fig. 2(a)]. We note that $Q$ factors as high as $10^{7}$ can be reached for smaller levitated magnetic particles [19]. By exciting certain mechanical modes of the levitated magnetic mirror, we examine how the dissipation rates of the resonant modes change. The excitation is achieved by modulating the current inside the driving coil above the levitated magnetic mirror. Figure 2(b) indicates that
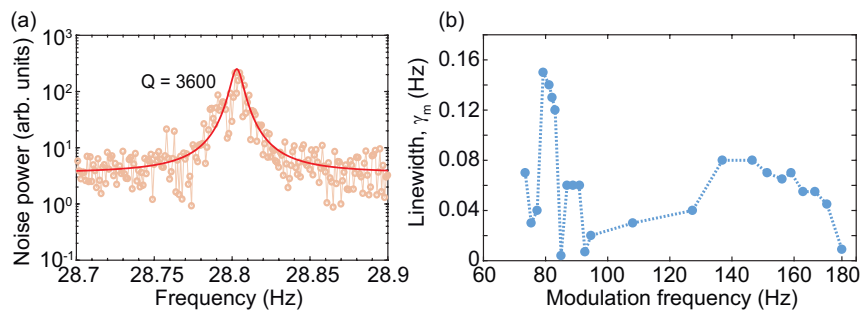

FIG. 2. (a) Spectrum of the coupled high- $Q$ mode $\omega_{v(x, \theta)}$ measured with a resolution bandwidth of $0.001 \mathrm{~Hz}$. (b) The fitted linewidth as a function of the external modulation frequency with a constant modulation amplitude $\Delta B=28 \mu \mathrm{T}$ shows the effect of external excitation on mechanical $Q$. The dotted line here is a guide to the eye.

the linewidths of the resonant modes dramatically increase as the modulation frequency approaches the resonant frequencies of the levitated magnetic mirror. This also suggests that the ring-down measurement is not a reliable way to measure $Q$ as the excitation itself lowers the $Q$.

The origin of this excitation-induced dissipation is nonlinear damping that can be used to reduce the vibrational noise of certain modes. When the levitated magnetic mirror oscillates above the SD, its motion modulates the stray magnetic field on the surface of the SD. This is the result of the inhomogeneous magnetic field sensed on the SD surface when the mirror moves. This forces the superconducting trapped magnetic vortices to undergo a dynamical variation of configuration or density. The dissipation arising from this transformation is nonlinear. The dynamic dissipation energy during a hysteretic cycle per unit volume, $\delta E$, can be described using a modified Bean's critical state model or Irie-Yamafuji model [21-23] that follows the following relationship:

$$
\frac{\delta E-\delta E_{0}}{\delta E_{0}} \simeq \frac{1}{2} \omega \tau_{0}
$$

where $\delta E_{0}$ is the static energy loss per cycle per unit volume and $\tau_{0}$ is the timescale of the oscillation. In the regime where $\omega \tau_{0}>1$, the dynamic dissipation can exceed the static one. Similar results are expected using the collective weak-pinning theory [24] where the crossover between the hysteretic and viscous damping regimes can be calculated.

In the present case, when the vertical mode $\left(\omega_{z}\right)$ is excited, $\delta B$ along the orthogonal directions [e.g., $\left.\omega_{u(x, \theta)}\right]$ also changes, which introduces vortex-vortex interaction leading to nontrivial dissipation of the flux motion [25]. This effect can then increase the damping of the orthogonal modes without increasing their vibrational amplitude. In the thermal noise limit, the mode temperature can be calculated using the cold damping theory [26]. To obtain a theoretical description of damping the vibrational noise of the levitated magnetic mirror, we expand on the theory of feedback cooling [27-29] and use the appropriate description of forces and external excitations to derive the limits of vibrational noise reduction. The viscous force driving the levitated magnetic mirror with resonant frequency, $\omega_{m}$, and amplitude, $A$, can be written as

$$
F_{v}(t)=-\frac{2 \delta E_{0} V}{A^{2} \omega_{m}} \dot{u}(t)
$$



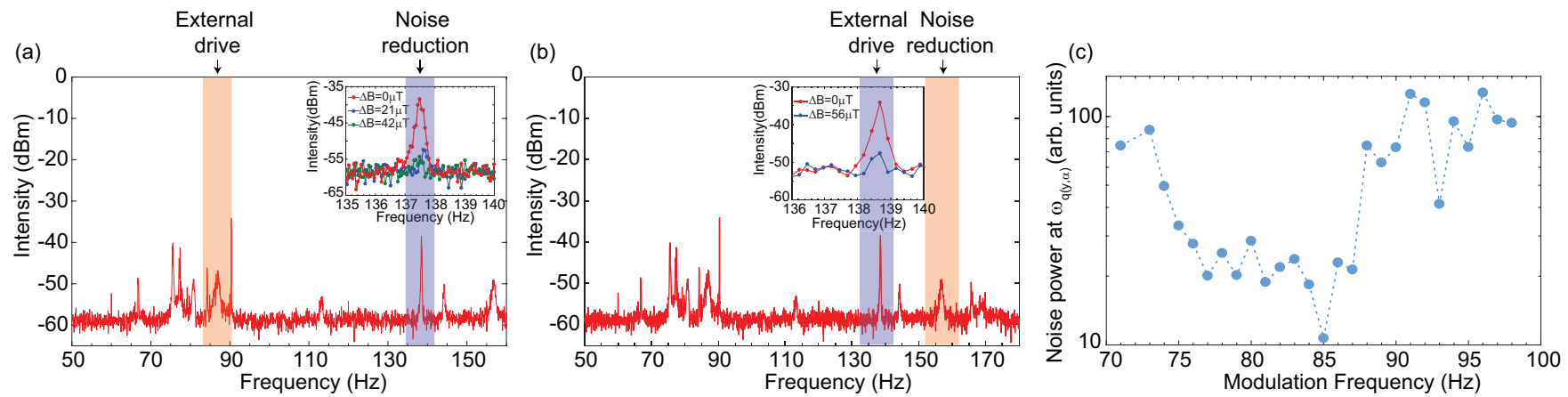

FIG. 3. [(a) and (b)] Damping of mechanical vibrations at $\omega_{q(y, \alpha)} \simeq 138 \mathrm{~Hz}$ via resonant excitation of $\omega_{z}$ and $\omega_{u(x, \theta)}$ modes, respectively. Inset shows the damped mode when different magnetic excitation amplitudes $(\Delta B)$ are applied. (c) The normalized vibrational intensity noise of $\omega_{q(y, \alpha)}$ as a function of modulation frequency for the fixed $\Delta B=56 \mu \mathrm{T}$ applied to the driving coil. The dotted line is a guide to the eye.

where $V$ is the volume of the SD where the dissipation occurs. The viscous force spectrum then becomes $F_{v}(\omega)=$ $K(\omega) u(\omega)$, where $K(\omega)=-i 2 \delta E_{0} V \omega /\left(A^{2} \omega_{m}\right)$. Assuming that the driven mode (where the external modulation force is parked) is far detuned from the damped mode (the amplitude fluctuation caused by the off-resonant drive, $\delta u \ll 1$ ), the effective mode temperature can be calculated to be $T_{\text {eff }} \simeq \frac{T}{1+\beta}$, where $\beta=2 \delta E_{0} V /\left(m A^{2} \omega_{m} \gamma_{m}\right), \gamma_{m}$ and $m$ are the linewidth of the damped mode and mass of the levitated mirror, respectively. In this case, the effective linewidth of the damped or cooled mode can be written as $\gamma_{\text {eff }}=(1+\beta) \gamma_{m}$. In practice, the residual excitation of the damped mode limits the lowest mode temperature that can be achieved using the nonlinear damping mechanism. The modified effective mode temperature then becomes $T_{\text {eff }}=\frac{T}{1+\beta}+T_{0}$, where $T_{0} \simeq \frac{\beta^{2}}{1+\beta} \eta_{\mathrm{ex}} T$ and $\eta_{\mathrm{ex}}$ is the ratio of the residual excitation energy to the thermal energy.

Figure 3 shows the result of noise suppression of one vibrational mode by driving another resonant mode. To the best of our knowledge, this is the first time such a controlled driven dissipation phenomenon has been reported. We have observed that the oscillation amplitude of the mode at $\omega_{q(y, \alpha)}=138 \mathrm{~Hz}$ is suppressed when the external magnetic field excitation is resonant with either the vertical oscillation mode of the mirror $\left(\omega_{z}=85 \mathrm{~Hz}\right)$ or the coupled mode $\omega_{u(x, \theta)}=158 \mathrm{~Hz}$ [see Figs. 3(a) and 3(b)]. As the magnetic modulation $(\Delta B)$ amplitude of the external excitation increases, the mechanical vibrational intensity of the coupled mode $\omega_{q(y, \alpha)}$ is suppressed by $>17 \mathrm{~dB}$. Figure $3(\mathrm{c})$ shows the normalized intensity of the coupled mode $\omega_{q(y, \alpha)}$ as a function of modulation frequency. We have observed no clear damping effect when the modulation frequency is more than a few $\mathrm{Hz}$ away from the resonance frequency. Moreover, we have also observed that the vibrational noise of multiple modes $\left[\omega_{q(y, \alpha)}\right.$ and $\left.\omega_{u(x, \theta)}\right]$ can be simultaneously suppressed, although with different efficiencies, by exciting the vertical mode. At elevated background excitations (e.g., seismic noise), the increased damping reduces the mechanical $Q$ factors [see Fig. 2(b)]. This effect has also been observed with superconducting levitated magnetic particles [19]. Results of Figs. 4(a) and 4(b) were obtained in an experimental run where the mechanical modes show lower $Q$ s. Here we also observe the similar controlled driven dissipation phenomenon as in Fig. 3. The low- $Q$ vibrational spectrum in Fig. 4(a) enables us to accurately determine the linewidth and energy of $\omega_{q(y, \alpha)}$, which is no longer limited by the resolution bandwidth of our measurement. Figure 4(b) shows that the fitted experimental linewidth of $\omega_{q(y, \alpha)}$ linearly increases with the magnetic field modulation amplitude $(\Delta B)$. The linear change was also predicted by the collective weak-pinning theory [24]. As $\Delta B$ increases from 0 to $70 \mu \mathrm{T}$, the vibrational noise power of the coupled mode $\left[\omega_{q(y, \alpha)}\right]$
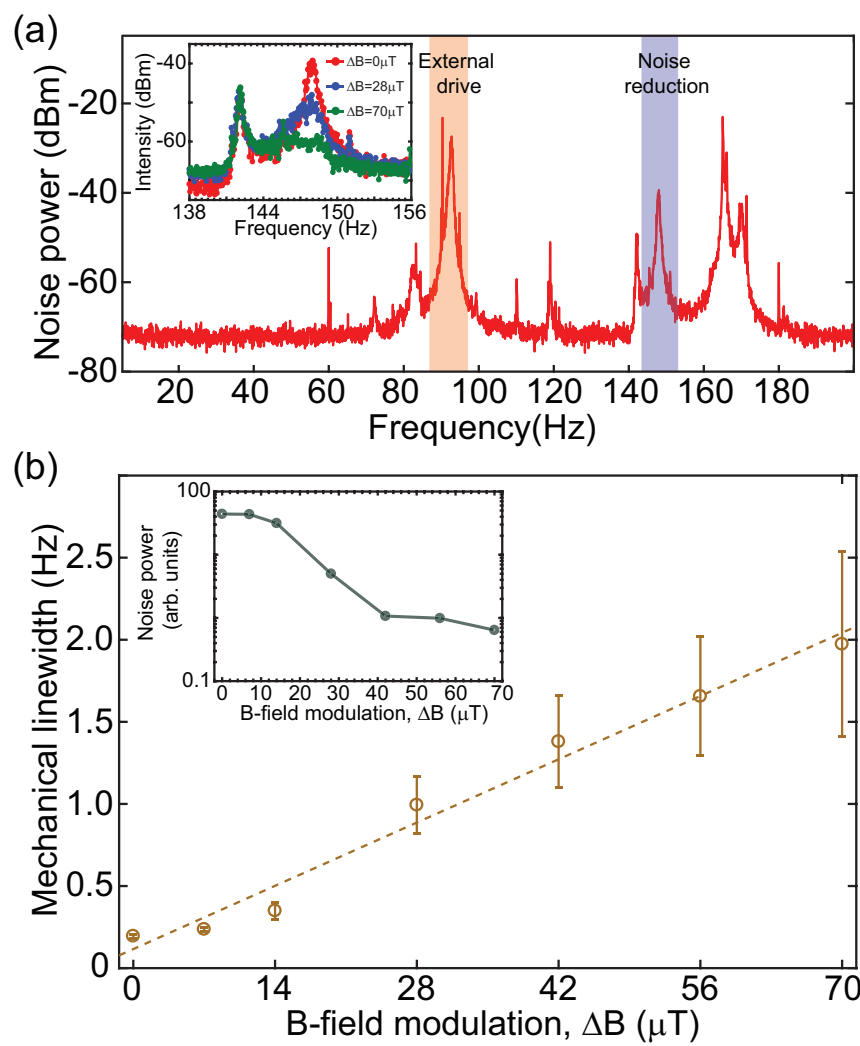

FIG. 4. (a) The vibrational spectrum with the controlled driven and damped modes indicated at $\omega_{z}$ and $\omega_{q(y, \alpha)}$, respectively. Inset shows the damped mode $\left[\omega_{q(y, \alpha)}\right]$ for different external magnetic modulation amplitudes $(\Delta B)$ induced by the current modulation in the coil. (b) The measured linewidth of $\omega_{q(y, \alpha)}$ mode as a function of $\Delta B$. The inset shows the normalized noise power of $\omega_{q(y, \alpha)}$ mode as a function of $\Delta B$. The line in the inset is a guide to the eye. 
reduces by about two orders of magnitude while its linewidth increases by an order of magnitude. From Fig. 4(b), we estimate $\beta=10 \pm 2$. Also, using the equipartition theorem, we can calculate an effective noise reduction factor of 10 , in agreement with $\beta \simeq 10$ obtained from the low- $Q$ mechanical modes [Fig. 4(a)]. In experimental runs where $\omega_{q(y, \alpha)}$ mode shows higher $Q$ ( $>4$ times higher), the effective noise reduction factor obtained is about 60 [Fig. 3(a)]. This again agrees with the theory described above, suggesting that $\beta$ is inversely proportional to $\gamma_{m}$. In our experiment, we note that the mechanical vibrations of the superconducting levitated magnetic mirror are not thermal-noise limited since the seismic noise is $>4$ orders of magnitude higher than the thermal noise in the frequency range of interest. Because of the broadband nature of the seismic noise over the frequency range of interest, we assume the cold damping theory can still be used to model the damping or cooling process by assuming an effective mode temperature. More investigations at lower background noise are needed to verify the cooling effect at the thermalnoise limit.

The maximum noise reduction factor is limited by the off-resonant excitations and residual heating due to the coupling to the externally excited modes. The geometry of the levitated magnetic mirror defines the strength of the inhomogeneity and mode coupling in the system, thus playing an important role in the selective mode damping process. The magnetic mirror with diametrical magnetization used in our experiment provides the asymmetry needed to have distinct mechanical modes. This is accompanied by an inhomogeneous field arising from the oscillating magnetic mirror. While large inhomogeneity enhances the damping process for certain modes, the low inhomogeneity of certain oscillations gives rise to ultra-high- $Q$ resonances in the system [19]. Our experiment helps to better understand the nature of mode coupling and nonlinear dissipation and suggests a way to use such mechanisms to cool the vibrational noise at certain modes by heating or exciting other modes. Precision magnetic sensing [11,30], coherent magnetic-optical trapping [31-33], and ground-state cooling of microscale magnets [13] are among the applications of superconducting levitation studied here. An in-depth theoretical understanding of off-resonance dissipation in multi-mode systems is needed to guide future experiments designed for ultrahigh $Q$ and/or low-noise superconducting levitation.

In conclusion, we study mode coupling and dissipation mechanisms in a levitated magnetic mirror above a superconductor. We model vibrational modes of the mirror and test mode coupling between different degrees of freedom of the levitated object. We observe that the nonlinear dissipation in superconducting levitation systems could be harnessed to control and damp certain mechanical modes. We show that via the excitation of certain modes, the nonlinear damping can be used to suppress the thermal/mechanical vibrational noise in the orthogonal modes. In this way, a maximum noise reduction factor of about 60 was achieved. To the best of our knowledge, this is the first time such a controlled driven dissipation mechanism is reported. As such method does not rely on the signal-to-noise ratio of detection (as is the case for feedback cooling), the approach can enable cooling certain modes independent of their detection and position in the spectrum.

This work was in part supported by Purdue University and the Air Force Office of Scientific Research (AFOSR), Grant No. FA 9550-19-1-0371.
[1] C. Gonzalez-Ballestero, M. Aspelmeyer, L. Novotny, R. Quidant, and O. Romero-Isart, Levitodynamics: Levitation and control of microscopic objects in vacuum, Science $\mathbf{3 7 4}$, eabg3027 (2021).

[2] J. Chan, T. M. Alegre, A. H. Safavi-Naeini, J. T. Hill, A. Krause, S. Gröblacher, M. Aspelmeyer, and O. Painter, Laser cooling of a nanomechanical oscillator into its quantum ground state, Nature (Lond.) 478, 89 (2011).

[3] G. S. MacCabe, H. Ren, J. Luo, J. D. Cohen, H. Zhou, A. Sipahigil, M. Mirhosseini, and O. Painter, Nano-acoustic resonator with ultralong phonon lifetime, Science 370, 840 (2020).

[4] A. B. Shkarin, A. D. Kashkanova, C. D. Brown, S. Garcia, K. Ott, J. Reichel, and J. G. E. Harris, Quantum Optomechanics in a Liquid, Phys. Rev. Lett. 122, 153601 (2019).

[5] A. M. Kaufman, B. J. Lester, and C. A. Regal, Cooling a Single Atom in an Optical Tweezer to Its Quantum Ground State, Phys. Rev. X 2, 041014 (2012).

[6] J. Hu, A. Urvoy, Z. Vendeiro, V. Crépel, W. Chen, and V. Vuletić, Creation of a bose-condensed gas of $87 \mathrm{rb}$ by laser cooling, Science 358, 1078 (2017).

[7] U. Delić, M. Reisenbauer, K. Dare, D. Grass, V. Vuletić, N. Kiesel, and M. Aspelmeyer, Cooling of a levitated nanoparticle to the motional quantum ground state, Science 367, 892 (2020).
[8] M. Cirio, G. K. Brennen, and J. Twamley, Quantum Magnetomechanics: Ultrahigh-Q-Levitated Mechanical Oscillators, Phys. Rev. Lett. 109, 147206 (2012).

[9] O. Romero-Isart, L. Clemente, C. Navau, A. Sanchez, and J. I. Cirac, Quantum Magnetomechanics with Levitating Superconducting Microspheres, Phys. Rev. Lett. 109, 147205 (2012).

[10] J. Gieseler, A. Kabcenell, E. Rosenfeld, J. D. Schaefer, A. Safira, M. J. A. Schuetz, C. Gonzalez-Ballestero, C. C. Rusconi, O. Romero-Isart, and M. D. Lukin, Single-Spin Magnetomechanics with Levitated Micromagnets, Phys. Rev. Lett. 124, 163604 (2020).

[11] X. Jiang, J. Rudge, and M. Hosseini, Superconducting levitation of a mg-scale cavity mirror, Appl. Phys. Lett. 116, 244103 (2020).

[12] C. Timberlake, G. Gasbarri, A. Vinante, A. Setter, and H. Ulbricht, Acceleration sensing with magnetically levitated oscillators above a superconductor, Appl. Phys. Lett. 115, 224101 (2019).

[13] K. Streltsov, J. S. Pedernales, and M. B. Plenio, Ground-State Cooling of Levitated Magnets in Low-Frequency Traps, Phys. Rev. Lett. 126, 193602 (2021).

[14] J. Druge, C. Jean, O. Laurent, M.-A. Méasson, and I. Favero, Damping and non-linearity of a levitating magnet in rotation above a superconductor, New J. Phys. 16, 075011 (2014). 
[15] T. Sugiura, T. Inoue, and H. Ura, Nonlinear vibration of a coupled high-tc superconducting levitation system, Physica C: Superconductivity 412, 778 (2004).

[16] T. Sugiura, T. Aoyagi, H. Ura, and M. Yoshizawa, Parametrically excited horizontal and rolling motion of a levitated body above a high-t/sub c/superconductor, IEEE Trans. Appl. Supercond. 13, 2247 (2003).

[17] J. R. Hull and A. Cansiz, Vertical and lateral forces between a permanent magnet and a high-temperature superconductor, J. Appl. Phys. 86, 6396 (1999).

[18] T. Sugiura and Y. Uematsu, Analysis of electromagnetic force and torque in high-tc superconducting levitation based on the advanced mirror image method, Nihon Kikai Gakkai Ronbunshu, C Hen/Trans. Jpn. Soc. Mech. Eng. C 66, 1138 (2000).

[19] A. Vinante, P. Falferi, G. Gasbarri, A. Setter, C. Timberlake, and H. Ulbricht, Ultralow Mechanical Damping with MeissnerLevitated Ferromagnetic Microparticles, Phys. Rev. Appl. 13, 064027 (2020).

[20] L. M. Manojlović, Quadrant photodetector sensitivity, Appl. Opt. 50, 3461 (2011).

[21] T. Matsushita et al., Flux Pinning in Superconductors, Vol. 164 (Springer, Berlin, 2007).

[22] F. Irie and K. Yamafuji, Theory of flux motion in non-ideal typeii superconductors, J. Phys. Soc. Jpn. 23, 255 (1967).

[23] C. P. Bean, Magnetization of high-field superconductors, Rev. Mod. Phys. 36, 31 (1964).

[24] D. B. Liarte, D. Hall, P. N. Koufalis, A. Miyazaki, A. Senanian, M. Liepe, and J. P. Sethna, Vortex Dynamics and Losses Due to Pinning: Dissipation from Trapped Magnetic Flux in Resonant Superconducting Radio-Frequency Cavities, Phys. Rev. Appl. 10, 054057 (2018).

[25] V. Sokolovsky, V. Meerovich, M. Spektor, G. A. Levin, and I. Vajda, Losses in superconductors under non-sinusoidal currents and magnetic fields, IEEE Trans. Appl. Supercond. 19, 3344 (2009).

[26] M. Pinard, P.-F. Cohadon, T. Briant, and A. Heidmann, Full mechanical characterization of a cold damped mirror, Phys. Rev. A 63, 013808 (2000).

[27] T. Li, S. Kheifets, and M. G. Raizen, Millikelvin cooling of an optically trapped microsphere in vacuum, Nat. Phys. 7, 527 (2011).

[28] A. Vinante, M. Bignotto, M. Bonaldi, M. Cerdonio, L. Conti, P. Falferi, N. Liguori, S. Longo, R. Mezzena, A. Ortolan, G. A. Prodi, F. Salemi, L. Taffarello, G. Vedovato, S. Vitale, and J. P. Zendri, Feedback Cooling of the Normal Modes of a Massive Electromechanical System to Submillikelvin Temperature, Phys. Rev. Lett. 101, 033601 (2008).

[29] G. Guccione, Optomechanical Enhancements for Applications in Metrology, Ph.D. thesis, Department of Quantum Science, Research School of Physics and Engineering, The Australian National University, 2017.

[30] J. R. Maze, P. L. Stanwix, J. S. Hodges, S. Hong, J. M. Taylor, P. Cappellaro, L. Jiang, M. G. Dutt, E. Togan, A. Zibrov et al., Nanoscale magnetic sensing with an individual electronic spin in diamond, Nature (Lond.) 455, 644 (2008).

[31] G. Guccione, M. Hosseini, S. Adlong, M. T. Johnsson, J. Hope, B. C. Buchler, and P. K. Lam, Scattering-Free Optical Levitation of a Cavity Mirror, Phys. Rev. Lett. 111, 183001 (2013).

[32] B. L. Brown, A. J. Dicks, and I. A. Walmsley, Coherent Control of Ultracold Molecule Dynamics in a Magneto-Optical Trap by Use of Chirped Femtosecond Laser Pulses, Phys. Rev. Lett. 96, 173002 (2006).

[33] H. J. Williams, L. Caldwell, N. J. Fitch, S. Truppe, J. Rodewald, E. A. Hinds, B. E. Sauer, and M. R. Tarbutt, Magnetic Trapping and Coherent Control of Laser-Cooled Molecules, Phys. Rev. Lett. 120, 163201 (2018). 